\title{
A Methodology for Conceptual Design of Mechanisms by Parsing Design Specifications
}

\author{
Dar-Zen Chen ${ }^{1}$ \\ Professor \\ e-mail: dzchen@ntu.edu.tw \\ Wei-Ming Pai \\ Graduate Assistant \\ Department of Mechanical Engineering, \\ National Taiwan University, \\ Taipei, Taiwan 10660, Republic of China
}

In this paper, design specifications for the conceptual design of mechanisms are parsed into three coherent categories: functional requirements, structural requirements, and design constraints. Based on functional requirements, the functioning kinematic chain of a mechanism can be constructed by connecting the functioning links accordingly. From structural requirements, admissible kinematic structures are searched from the existing atlases of kinematic structures. Those admissible kinematic structures, which comprise at least one segment with the same characteristics of the functioning kinematic chain, are identified as compatible kinematic structures. Thus, feasible mechanisms that fulfill design specifications can be yielded by labeling joints in the compatible kinematic structures subject to design constraints. With this methodology, classified design specifications are used to guide designer through various stages of the conceptual design process in a systematic manner. The creation of the variable-stroke engine mechanism [Freudenstein, F., Maki, E. R., 1983, "Development of an Optimum Variable-Stroke Internal-Combustion Engine Mechanism From the Viewpoint of Kinematic Structure," ASME J. Mech., Trans., Automat., Des., Vol. 105, pp. 259-267] is used as an illustrative example.

[DOI: $10.1115 / 1.2044767]$

\section{Introduction}

In the conceptual design of mechanisms, initial comprehension of an object mechanism is solely based on a set of given design specifications, in which what a mechanism should do [1] is described. From these design specifications, certain significant structural characteristics of the mechanism, such as the degree-offreedom (DOF), the number of links, the nature of motion, and the number of independent loops, etc., are usually classified as structural requirements. Based strictly on structural requirements, Freudenstein and Maki [2] developed a systematic approach for the conceptual design of mechanisms, where admissible mechanisms are enumerated. Atlases of admissible kinematic structures, such as those of kinematic structures with up to six links [3,4], eight links [5,6], ten links [7-11], and eleven links [12] have been established during the past three decades. Following the enumeration of admissible kinematic structures, ground link is assigned and joint types are labeled in as many nonisomorphic ways as possible in search of candidate mechanisms. Since only structural requirements are used to enumerate candidate mechanisms in this approach, the needs for human ingenuity and experience involved can be substantially alleviated. Functional requirements together with design constraints are then used for further viability evaluation. As a result, feasible mechanisms that fulfill design specifications are obtained by the way of rejecting those candidate mechanisms failed in the evaluation process. Figure 1 shows the procedure of this approach. This approach had been a major breakthrough in the pursuit of systematic approaches for the conceptual design of mechanisms, and had been widely applied to a variety of applications [13-20]. However, numerous infeasible mechanisms are subsumed since only structural requirements are considered in the enumeration of candidate mechanisms. Thus, in turn, it leads to circumstances, where infeasible mechanisms need to be carefully screened out from enumerated candidate mechanisms, resulting in a tedious and inefficient process.

Corresponding author.

Contributed by the Mechanisms and Robotics Committee for publication in the Journal of Mechanical Design. Manuscript received June 3, 2004; revised March 8, 2005. Associate Editor: G. R. Pennock.
In this paper, a systematic methodology for the conceptual design of mechanisms is presented. As shown in Fig. 2, design specifications are classified as functional requirements, structural requirements and design constraints. Motion requirements of a set of functioning links, such as the ground, input and output links, are specified as functional requirements. Mapping functional requirements to the connections of functioning links, the functioning kinematic chain of a mechanism can be constructed. On the other hand, based on structural requirements, admissible kinematic structures of the mechanism are searched from the existing atlases of kinematic structures. By assigning the functioning kinematic chain into admissible kinematic structures subject to design constraints on locations of functioning links, compatible kinematic structures can be identified. According to design constraints on joints of the mechanism, joints in those compatible kinematic structures are labeled to yield feasible mechanisms. With this procedure, conceptual design of mechanisms can be performed in an efficient manner. The creation of the variable-stroke engine mechanism [13] is used as an example to demonstrate the methodology.

\section{Classification of Design Specifications}

Design specifications with various natures can be identified and classified into three coherent categories: functional requirements, structural requirements, and design constraints. Pahl and Beitz [21] defined functional requirements as "the general input/output relationship of a mechanism whose purpose is to perform a task." Suh [22] deemed functional requirements the desired output of a mechanism. However, few developed systematic approach for the conceptual design of mechanisms starts with the functional requirements since they are generally established in terms of a descriptive form. Hence, the embodiment process for such descriptive formulations usually depends on the ingenuity, intuition and experience of a designer, and appears to be the most challenging task in the conceptual design stage.

In general, functional requirements mandate the motion relations of a set of specific functioning links, such as the input, output, and ground, etc. Motions of input/output links are ordinarily specified, and the operating direction of input/output links 


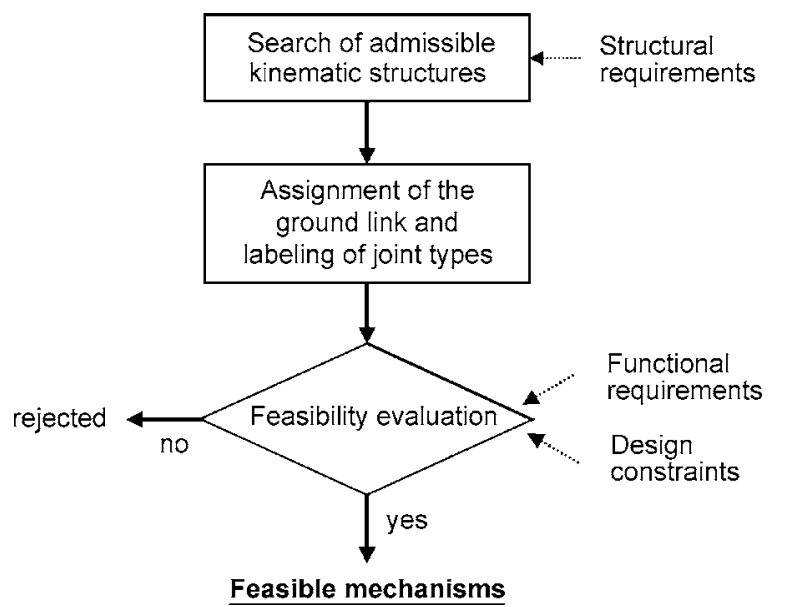

Fig. 1 Conceptual design approach by Freudenstein and Maki [2]

with respect to the ground link, etc. is often described. The required motions between these links are associated with the motion type and the operating direction. Reuleaux [23] recognized that the yield of required motions between links depends on their connections. Proper adjacency arrangements for the functioning links of a mechanism yields desired motion potentially. The number of links connected to one link is referred to the connectivity of links. A link with higher connectivity implies more constraints on its motion. The types of joints between links determine the motion type of links, and the orientation of joints determines the operating direction of links. Hence, functional requirements can be mapped into the required motion of functioning links and the required motion of functioning links can be embodied as (1) adjacencies of functioning links, (2) connectivity of functioning links, and (3) type and/or orientation of joints between functioning links.

For the variable-stroke engine mechanism, design specifications developed by Freudenstein and Maki [13] are listed in Tables 1(a) and $1(b)$. It can be seen that design specifications $\mathbf{S 4}, \mathbf{S 5}, \mathbf{S 6}, \mathbf{A 2}$, and $\mathbf{A 3}$ specify required motions of a set of specific links, including the piston, ground, connecting rod, and crank. Thus, these design specifications are classified as functional requirements, and these four links are identified as functioning links of the variable-

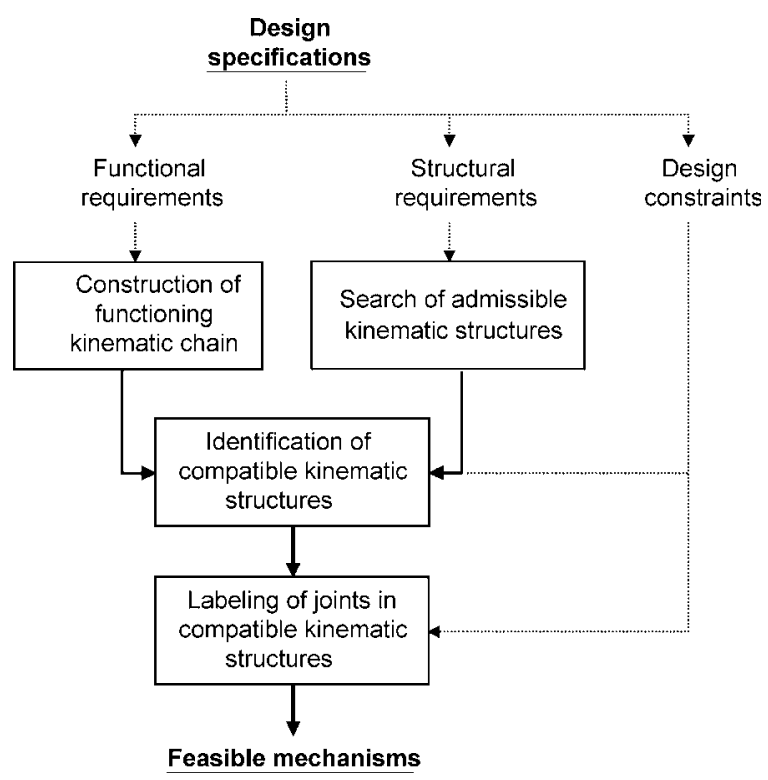

Fig. 2 Proposed conceptual design approach
Table 1 (a) Design specifications of the engine mechanism: search specifications [13]; (b) Design specifications of the engine mechanism: evaluation criteria [13]

\section{(a)}

S1. The search is limited to plane six-link mechanisms with seven joints and plane eight-link mechanisms with 10 joints (two and three independent loops); all joints being turning pairs $(R)$ or straight-line sliding pairs $(P)$ and all mechanisms obeying the general degree-of-freedom equation (single-degree-of-freedom)

s2. The maximum number of sliding pairs cannot exceed two (this limits the amount of sliding in the mechanism).

S3. No link can have more than one sliding joint (again this limits the degree of sliding in the mechanism).

S4. Only the piston can be ground-connected by a sliding pair. This precludes the possibility of links other than the piston being in pure translation. Such a condition would be awkward and probably substantially increase frictional resistance in the mechanism.

S5. The ground link of all six-link structures should be ternary (i.e., must have three joints) since control via a floating-link connection is considered undesirable.

S6. All eight-link structures, which do not have at least one link with four joints, are excluded This limits the number of floating links (i.e. not connected to the ground link) to three as is desirable for dynamics and balance.

S7. All eight-link structures with only one sliding pair are eliminated. The reason for this restriction is as follows: Variable displacement with constant compression ratio can be obtained by (a) chaning the length of a ground link; and (c) combinations of these. Possibilities (a) and (c) are rejected because it is desirable to have a grounded control. If possibility (b) is to be realized with a mechanism having only turning pairs and one sliding pair (piston-to-ground connection), this leads to poor transmission angles, unfavorable force transmission, large acceleration fluctuations large size, or combinations of these. If, however, a sliding pair is part of the stroke adjustment and the mechanism proportioned so that there is minimal sliding at this point, these circumstances do not, in general, apply

S8. Controls to vary the displacements are not included in the structures which are enumerated in the search

\section{(b)}

A1. No sliding pair may exist in the drive loop (i.e., the loop which contains the crank) so that sliding in this high-speed loop is eliminated.

A2. The piston must be connected to a binary (i.e., two-jointed) connecting rod, because a connecting rod with more than two joints is likely to develop excessive side-thrust and lead to high piston friction.

A3. The crank. which is ground-connected by a turning pair, must be binary (i.e., two jointed). This restriction may not be absolutely essential, but will avoid complicated cranks and likelihood of high piston side-thrust.

A4. The crank cannot be directly connected to the connecting rod fotherwise it would be very difficult to vary the stroke)

A5. The crank cannot be part of the control loop (i.e., the loop which is connected to stroke adjustment) because it would be difficult to design the control loop and there is the additional likelihood of excessive sliding and joint forces.

A6. Stroke variation cannot be achieved at the expense of requiring variation of the proportions of the links in the drive loop. Such variation would lead to large acceleration fluctuations and unfavorable dynamic and balance conditions.

stroke engine mechanism. The functional requirements can be further comprehended as the connections of the functioning links as follows.

The adjacencies of functioning links can be deduced from $\mathbf{S 4}$, $\mathbf{A 2}$, and $\mathbf{A} \mathbf{3}$ as shown in Table 2(a), where the adjacency between the piston and ground link is specified as $\mathbf{C 1}$, the adjacency between the piston and connecting rod as C2, and the adjacency between the crank and ground link as $\mathbf{C 3}$. The connectivity of these functioning links can be obtained from S5, S6, A2, and A3 as shown in Table 2(a). The connectivity of the ground link for six-link kinematic structures is specified as $\mathbf{C 4}$, the connectivity of the ground link for eight-link kinematic structures as $\mathbf{C 5}$, the connectivity of the connecting rod as $\mathbf{C 6}$, and the connectivity of the crank as C7. The type of the joints between the functioning links can also be obtained from $\mathbf{S 4}$ and $\mathbf{A 3}$ as shown in Table 2(a). The joint between the piston and ground link is specified as $\mathbf{C 8}$, and the joint between the crank and ground link as $\mathbf{C 9}$.

Design specifications with a set of parameters used to determine the kinematic structure of the mechanism are referred to structural requirements. This set of parameters include the DOF of 
Table 2 (a) Functional requirements of the variable-stroke engine mechanism; (b) Structural requirements of the variablestroke engine mechanism; (c) Design constraints of the variable-stroke engine mechanism

(a)

\begin{tabular}{l} 
Adjacencies of functioning links: \\
C1: The piston is adjacent to the ground link. (S4) \\
C2: The piston is adjacent to a connecting rod. (A2) \\
C3: The crank is adjacent to the ground link. (A3) \\
Connectivity of functioning links: \\
C4: For six-link kinematic structures, the connectivity of ground link is three. (S5) \\
C5: For eight-link kinematic structures, the connectivity of ground link is four. (S6) \\
C6: The connectivity of connecting rod is two. (A2) \\
C7: The connectivity of crank is two. (A3) \\
Joint types between functioning links: \\
C8: The joint between the piston and the ground link is a prismatic joint. (S4) \\
C9: The joint between the crank and the ground link is a revolute joint. (A3) \\
\hline (b) \\
R1: The mechanism is of single DoF. (S1) \\
R2: The mechanism is confined to planar nature of motion, namely $\lambda$ equals three. (S1) \\
R3: The number of links is up to eight links. (S1) \\
R4: There are two or three independent loops. (S1) \\
R5: The type of joints is limited to revolute joints and prismatic joints only. (S1) \\
\hline (C) \\
Constraints on joints of the mechanism: \\
D3: A prismatic joint cannot be adjacent to another prismatic joint. (S3) \\
D4: There is no prismatic joint in the drive loop. (A1) \\
D5: The number of prismatic joints cannot exceed two. (S2) \\
D6: There is at least one prismatic joint in the control loop. (S7) \\
Constraints on locations of functioning links: \\
D1: The crank cannot be placed in the control loop. (A5) \\
Cr. 1: There are three loops in the mechanism. \\
Cr. 2: The crank and piston are included in two separate loops and each belongs to \\
its respective loop only.
\end{tabular}

the mechanism, the nature of motion, the number of links, the number of independent loops and admissible types of joints, etc.

For the design specifications shown in Table 1, S1 involve all above-mentioned parameters and can be transformed into the structural requirements as shown in Table 2(b), where the DOF of the mechanism is specified as $\mathbf{R} \mathbf{1}$, the nature of motion as $\mathbf{R 2}$, the number of links as R3, the number of independent loops as $\mathbf{R 4}$, and admissible types of joints as R5.

Design specifications based on particular engineering reasoning and imposing restrictions on the mechanism are considered as design constraints. Two kinds of constraints are often involved: (1) constraints on the location of link such as the input, output, and ground, etc. and (2) constraints on joints of the mechanism.

The design specifications S2, S3, S7, A1, A4, and A5, which limit the locations and types of joints, are considered as design constraints. The constraints on locations of the functioning links can be obtained from A4 and $\mathbf{A 5}$ as shown in Table 2(c), where D1 prohibits the crank to be included in the control loop that contains an adjustable pivot used to vary the stroke, and D2 prevents the crank from being adjacent to the connecting rod. In addition to the control loop, the mechanism must consist of a drive loop that contains the crank and an output loop that contains the piston. On occasion, it is considered acceptable while the drive loop includes the control function as the mechanism has only two loops. However, on the contrary, it is out of consideration while the output loop includes the control function, since high piston side-thrust would make it difficult to vary the stroke. Hence, from D1, the possibility of a loop with dual functions of drive and control is excluded. Thus, we have

Cr. 1: There are three loops in the mechanism.

This criterion ensures the mechanism to have independent drive loop, output loop and control loop. Note that Cr. 1 has many concise requirements on the number of independent loops than R4. Since both crank and piston shall not be included in control loop, neither the crank nor the piston can be shared by two neighboring loops. Thus, we have

Cr. 2: The crank and piston are included in two separate loops and each belongs to its respective loop only.

From D2, the crank and the connecting rod are restrained from being connected together. Thus, we have

Cr. 3: The crank and connecting rod are not adjacent.

The constraints on the joints of the mechanism can be obtained from S2, S3, S7, and A1 as shown in Table 2(c). The constraint on the location of prismatic joints is specified as D3, the joints in the drive loop as D4, the number of prismatic joints in the mechanism as D5, and the number of prismatic joints in the control loop as D6.

From Table 1, it is observed that $\mathbf{S 8}$ and $\mathbf{A 6}$ do not belong to any of these three categories of design specifications. $\mathbf{S 8}$ limits the conceptual design on the creation of the variable-stroke engine mechanism itself, while A6 considers the dynamic and balance conditions of such a variable-stroke engine mechanism. Since S8 excludes the extra control mechanism used to vary displacement and $\mathbf{A 6}$ can only be evaluated at the final stage of the design, they are not considered in the conceptual design stage.

It can be seen that design specifications of mechanisms can be sequentially classified into functional requirements, structural requirements and design constraints. Note that design specifications, such as output displacement, velocity, acceleration, etc., which have no bearing on a design process until dimensional synthesis is implemented will not be considered here.

\section{Construction of Functioning Kinematic Chain(s)}

The construction of the functioning kinematic chain can be accomplished based on the adjacencies of functioning links, connectivity of the functioning links, and the joints types between functioning links deduced from functional requirements. Note that functioning links can constitute one or several kinematic chains in both open- or close-loop form and the connections of functioning links may not be sufficient to label all the joints in the kinematic chain(s).

The resulting kinematic chain(s) obtained represent(s) the functioning kinematic chain of the mechanism. The connectivity list of the functioning kinematic chain can be expressed as

$$
\mathrm{F}_{\mathrm{C}}:\left[\mathrm{C}_{1}, \mathrm{C}_{2} \ldots \mathrm{C}_{i} \ldots\right]
$$

where $\mathrm{C}_{i}$ is the connectivity of the $i$-th functioning link.

The joint DOF list of the functioning kinematic chain can be expressed as

$$
F_{D}:\left[D_{1}, D_{2} \ldots D_{j} \ldots\right]
$$

where $\mathrm{D}_{j}$ is the DOF of the joint between the $j$ th and $(j+1)$ th functioning link.

From C1, C2, and $\mathbf{C 3}$ of Table 2(a), it can be seen that the crank, ground, piston and the connecting rod are adjacent one by one. These four functioning links of the variable-stroke engine mechanism can thus be connected accordingly. Figure 3(a) shows the graph representation of the functioning kinematic chain. In graph representation of mechanisms [3], links are denoted by vertices and joints are denoted by edges, where the edge connection between vertices corresponds to the joint connection between links. In Fig. 3(a), the crank is denoted by a gray vertex, the ground link by double circles, the piston by a solid vertex, and the connecting rod by a rectangle. From $\mathbf{C 4}$ and $\mathbf{C 5}$, the connectivity of the ground link should be three for six-link kinematic structures and four for eight-link kinematic structures, as indicated in the parentheses shown in Fig. 3(b). From C6 and C7, both the connectivity of the connecting rod and the connectivity of the crank are two and identified in Fig. 3(b). The joints between the functioning links can be specified according to C8 and C9. As shown in Fig. 3(c), the joint between the piston and ground link is labeled as a prismatic joint $\mathrm{P}$ and the joint between the crank and ground link is labeled as a revolute joint R. Figure 3(c) shows the func- 


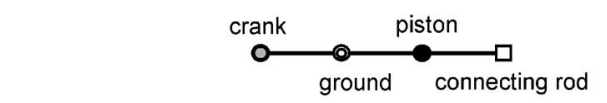

(a)
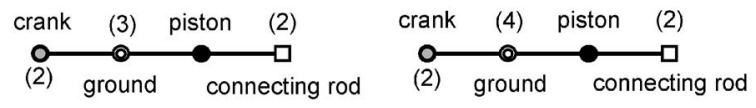

for six-link kinematic structures

for eight-link kinematic structures

(b)
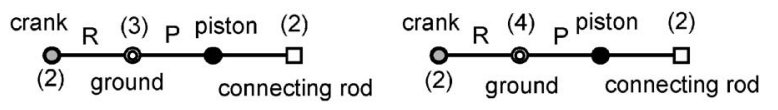

for six-link kinematic structures

\section{for eight-link} kinematic structures

(c)

\section{Fig. 3 Construction of the functioning kinematic chain}

tioning kinematic chain of the variable-stroke engine mechanism. The connectivity list of the functioning kinematic chain from the crank to the connecting rod is

$$
\mathrm{F}_{\mathrm{C}}:[2,3 \text {, any, 2] for six-link kinematic structures }
$$

and

$$
F_{C}:[2,4 \text {, any, 2] for eight-link kinematic structures }
$$

The joint DOF list of the functioning kinematic chain for sixlink and eight-link kinematic structures is

$$
\mathrm{F}_{\mathrm{D}}:[1,1,1]
$$

Through the construction of the functioning kinematic chain, functional requirements in descriptive form are successfully transformed into symbolic graph representation. The functioning kinematic chains shown in Fig. 3(c) carry necessary characteristics to fulfill the functional requirements. Hence, mechanism contains the functioning kinematic chain as part of it may be a potential candidate mechanism.

\section{Search for Admissible Kinematic Structures}

From the structural requirements, structure properties of the mechanism are specified and used in search of mechanisms where the functioning kinematic chain may be a part of them. The numbers of links and joints of a mechanism follow the general DOF equation as:

$$
\mathrm{DOF}=\lambda \cdot(n-1)-\sum_{i=1}^{\lambda-1}(\lambda-i) \cdot J_{i}
$$

where $\lambda$ is 3 for planar/spherical mechanisms, $\lambda$ is 6 for spatial mechanisms, and $J_{i}$ is the number of joints with $i$ DOF.

The number of joints is equal to the sum of joints with different DOF and can be written as

$$
J=\sum J_{i}
$$

For mechanisms with a given DOF and desired nature of motion, corresponding a number of links and number of joints, can be obtained by solving Eqs. (5) and (6). Kinematic structures with feasible numbers of links and joints can then be identified from the existing atlas of kinematic structures [3-12].

Substituting the DOF of the mechanism as one (R1) and $\lambda$ as three (R2) into Eq. (5), we have
Table 3 Graphs of kinematic structures with up to eight links [4]

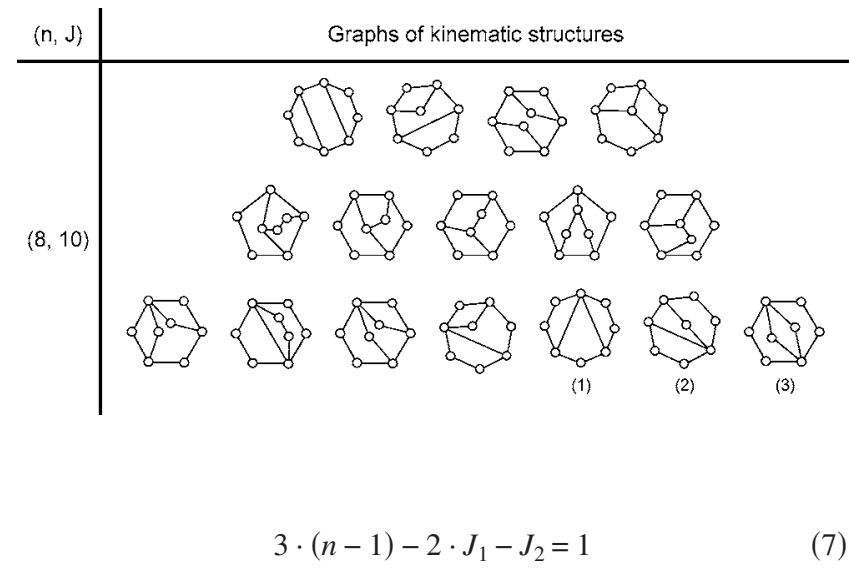

From Eq. (6), the number of joints can be expressed as

$$
J=J_{1}+J_{2}
$$

Since the joint type is confined to revolute and prismatic joints only (R5), the number of joints with two degrees of freedom, $J_{2}$, is set to zero. From Eq. (8), we have, the number of joints is the number of one DOF joints. Hence, Eq. (7) can be rewritten as

$$
J=(3 \cdot n-4) / 2
$$

According to Eq. (9), the number of joints $J$ can be solved for a given number of links $n$. Due to the number of joints is a positive integer and the number of links is up to eight (R3), the number of links and joints pair $(n, j)$ can be solved as $(4,4),(6,7)$, and $(8,10)$. These three sets of numbers of links and joints can be used to search for kinematic structures. Mruthyunjaya and Raghavan [7] enumerated kinematic structures with up to ten links, where one, two and sixteen kinematic structures are found for the number of links and joints pairs $(4,4),(6,7)$, and $(8,10)$, respectively. For the variable-stroke engine mechanism, since the number of independent loops is confined to three (Cr. 1), only the sixteen kinematic structures with eight links and ten joints fulfill this requirement. Table 3 shows graphs of these admissible kinematic structures.

\section{Identification of Compatible Kinematic Structures}

Since the admissible kinematic structures are obtained based on the structural requirements, they have to be carefully evaluated whether the functioning kinematic chain can be part of them. This indicates that kinematic structures, which comprise at least one segment with the same characteristics of the functioning kinematic chain, are considered as compatible kinematic structures. Hence, compatible kinematic structures can be identified, as long as there exists at least one path/circuit $[3,6]$ with the same connectivity list and joint DOF list of the functioning kinematic chain. This segment of the kinematic structures for the functioning kinematic chain can be identified with the design constraints on locations of the functioning links.

For the variable-stroke engine mechanism, the sixteen admissible kinematic structures will be checked whether at least one path with the connectivity list and joint DOF list match with Eqs. (3) and (4) for the functioning kinematic chain of eight-link kinematic structures. It can be seen that only those kinematic structures in the last row of Table 3 have a link with 4-connectivity and only the last six kinematic structures could comprise a path with the connectivity list of Eq. (3b). In this case, the check of the joint DOF list can be disregarded since all joints of the kinematic structures in Table 3 are of 1-DOF and will fulfill Eq. (4) spontaneously.

The criteria Cr. 1, Cr. 2, and Cr. 3 in Table 2(c) are used as a guide to further detect the location of paths for the functioning kinematic chain and identify compatible kinematic structures. 


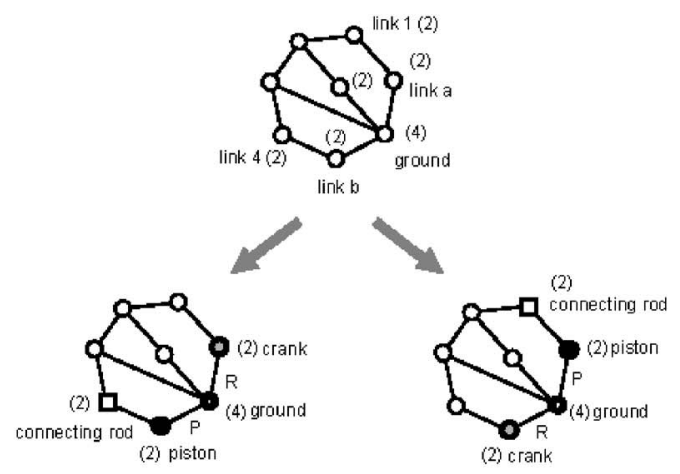

(a)
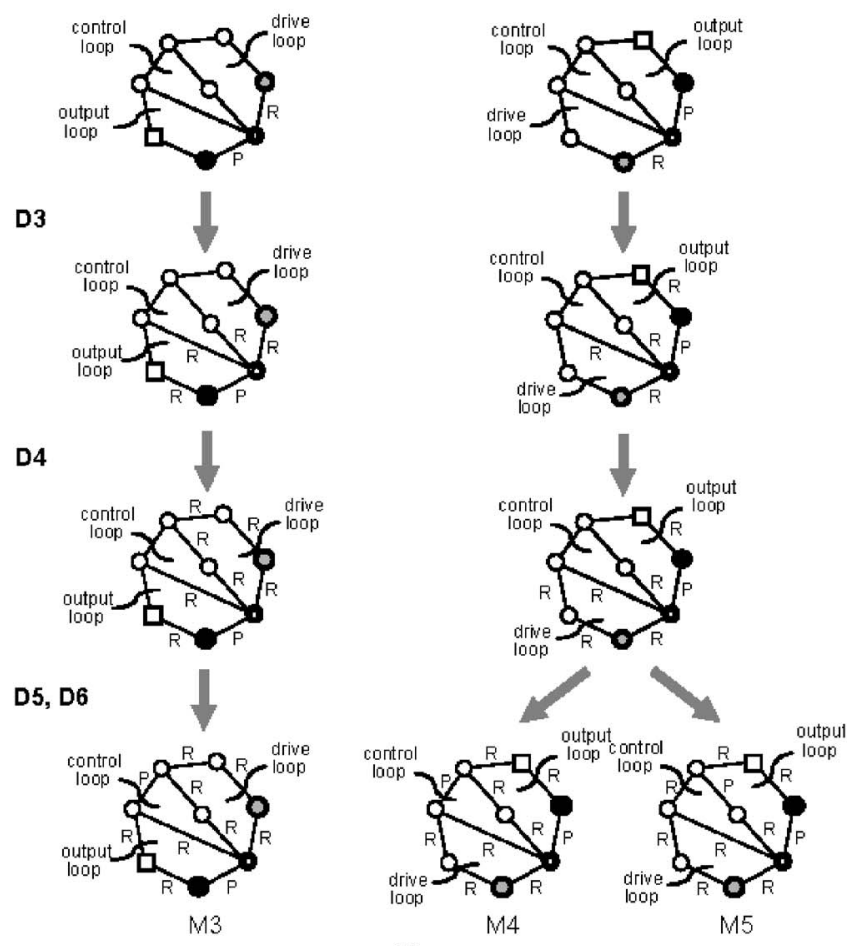

(b)

Fig. 4 (a) Identification of the functioning kinematic chain; (b) Labeling of unspecified joints

The second kinematic structure from the right in the last row of Table 3 is used as an example to illustrate how the functioning kinematic chain is identified in a kinematic structure as shown in Fig. 4(a). Locating the only one four-connectivity link as the ground link, it can be seen that only one of those two-connectivity links adjacent to the ground can be assigned as crank. However, from Cr. 2, the crank and piston shall be included in two separate loops and each belongs to its respective loops only. Hence, only link b can be selected as the piston for the case link a is assigned as the crank while link a can be selected as the piston for the case links $b$ is assigned as the crank. The joint between the crank and ground is labeled as revolute joint and the joint between the piston and ground is labeled as prismatic joint. Accordingly, two paths with the connectivity list $\mathrm{F}_{\mathrm{C}}$ : $[2,4,2,2]$ can be found for the functioning kinematic chain as shown in Fig. 4(a). Note that the two ends of these two paths, namely the crank and connecting rod are not adjacent and, thus, fulfill Cr. 3. With this process, the kinematic structures No. 1-3 in Table 3 are found to contain at least one such nonisomorphic path for the functioning kinematic chain, as shown in Table 4. Hence, kinematic structures No. 1-3
Table 4 Feasible variable-stroke engine mechanisms

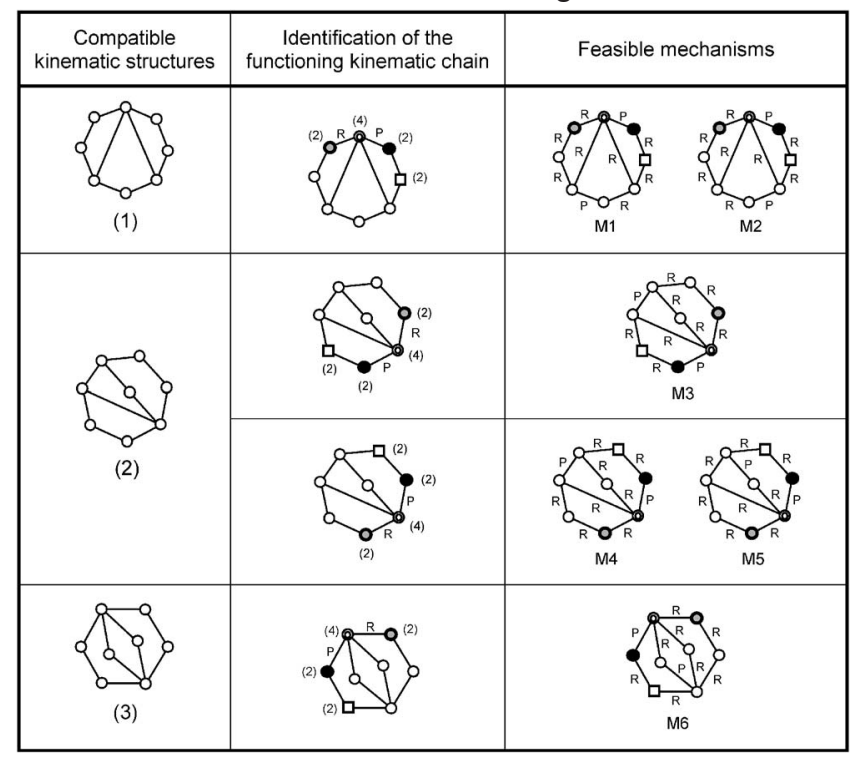

are identified as compatible kinematic structures.

It can be seen that by assigning the functioning kinematic chain into the searched kinematic structures, the number of compatible kinematic structures is substantially reduced. As per the approach of Freudenstein and Maki [2], all kinematic structures that fulfill structural requirements need to proceed to a cumbersome procedure by assigning the ground link and labeling joint types to every mechanism, feasible or nonfeasible. For example, according to Freudenstein and Maki's approach, hundreds of mechanisms can be enumerated [13], if all the possibility for the eighteen kinematic structures of Table 3 is explored. However, in this proposed methodology, only three of the eighteen kinematic structures are identified as compatible kinematic structure and, thus, the process on further implementation on joint labeling can be enormously simplified.

\section{Labeling of Joint in Compatible Kinematic Structures}

From the design constraints on the joints of the mechanism, limitations on the type, orientation, location, etc. of the joints are specified. Subject to these design constraints, unspecified joints of compatible kinematic structures are labeled to accomplish the enumeration of feasible mechanisms.

For the variable-stroke engine mechanism, all the compatible kinematic structures with identified functioning kinematic chain have ten joints, in which the joint between the ground link and piston and the joint between the ground link and crank have been labeled. The remaining eight joints are determined subject to D3, D4, D5, and D6 of Table 2(c). The two kinematic structures with identified functioning kinematic chain shown in Fig. 4(a) are used as an illustrative example, where the drive, output and control loop are further recognized in Fig. 4(b). From, D3, all unspecified joints adjacent to the prismatic joint $\mathrm{P}$ between the piston and ground are labeled as revolute joints $\mathrm{R}$ as shown in Fig. 4(b). By considering D4, unspecified joints in the drive loop are all labeled as R. In addition to the prismatic joint between the piston and ground, there should be another prismatic joint in the control loop to fulfill D5 and D6. As shown in Fig. 4(b), a feasible mechanism M3 is obtained by labeling the unspecified joint in the control loop as $\mathrm{P}$ and the other unspecified joints as R. Two feasible mechanisms M4 and M5 are obtained due to the two choices of unspecified joints in the control loop as $\mathrm{P}$ and the others as $\mathrm{R}$. With this process, as shown in Table 4, feasible mechanisms M1 
Table 5 Functional schemas of feasible variable-stroke engine mechanisms [13]

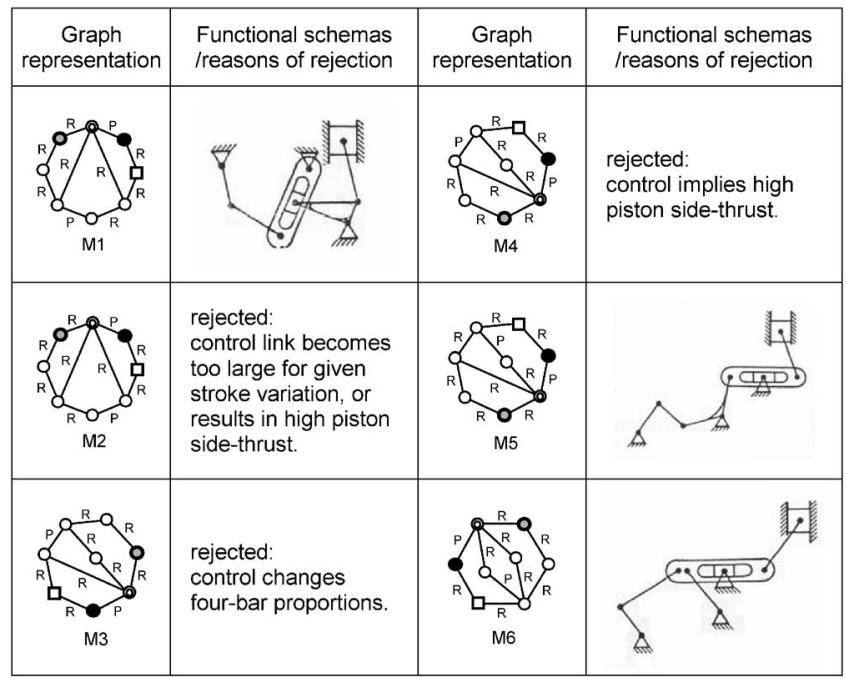

to M6 are enumerated based on the three compatible kinematic structures.

As per the study of Freudenstein and Maki [13], hundreds of enumerated kinematic structures are evaluated with functional requirements as well as other considerations from the experience of the designer. Three feasible mechanisms are obtained, i.e., M1, M5, and M6 shown in Table 5. It is found that mechanisms M2, M3, and M4 are considered infeasible in the study of Freudenstein and Maki [13] based mainly on subjective judgment. Functional schematic representation of the three feasible mechanisms M1, M5, and M6 and the reasons of Freudenstein and Maki [13] for rejecting M2, M3, and M4 are shown in Table 5.

\section{Conclusion}

In this paper, design specifications are classified as functional requirements, structural requirements, and design constraints. These different categories of design specifications are used to guide the construction of functioning kinematic chain, identification of compatible kinematic structures, and labeling of the joints in compatible kinematic structures. With this methodology, design specifications are well classified and systematically taken into account during the conceptual design process. As a result, the enumeration of feasible mechanisms is performed in a much more efficient manner. It is believed that this methodology can be beneficial for the design of mechanisms in the conceptual design stage.

\section{References}

[1] Ullman, D. G., 1992, The Mechanical Design Process, McGraw-Hill, New York.

[2] Freudenstein, F., and Maki, E. R., 1979, "The Creation of Mechanisms According to Kinematic Structure and Function," Environ. Plann. B, 6, pp. 375 391.

[3] Buchsbaum, F., and Freudenstein, F., 1970, "Synthesis of Kinematic Structure of Geared Kinematic Chains and Other Mechanisms," J. Mech., 5, pp. 357 392.

[4] Mayourian, M., and Freudenstein, F., 1984, "Development of an Atlas of The Kinematic Structures of Mechanisms," ASME J. Mech., Transm., Autom. Des., 106, pp. 458-461.

[5] Crossley, F. R. E., 1965, "The Permutations of Kinematic Chains of Eight Members or Less From the Graph-Theoretic Viewpoint," Developments in Theoretical and Applied Mechanics, Pergamon, Oxford, Vol. 2, pp. 467-486.

[6] Franke, R., 1958, Vom Aufbau der Getriebe, VDI Verlag, Dusseledrof, Vol I.

[7] Mruthyunjaya, T. S., and Raghavan, M. R., 1979, "Structural Analysis of Kinematic Chains and Mechanisms Based on Matrix Representation," ASME J. Mech. Des., 101, pp. 488-494.

[8] Mruthyunjaya, T. S., and Raghavan, M. R., 1984, "Computer-Aided Analysis of the Structure of Kinematic Chains," Mech. Mach. Theory, 19, pp. 357-368.

[9] Mruthyunjaya, T. S., 1984, “A Computerized Methodology for Structural Synthesis of Kinematic Chains: Part 1-Formulation," Mech. Mach. Theory, 19, pp. 487-495.

[10] Mruthyunjaya, T. S., 1984, “A Computerized Methodology for Structural Synthesis of Kinematic Chains: Part 2-Application to Several Fully or Partially Known Cases," Mech. Mach. Theory, 19, pp. 497-505.

[11] Mruthyunjaya, T. S., 1984, "A Computerized Methodology for Structural Synthesis of Kinematic Chains: Part 3-Application to the New Case of 10-Link, Three-Freedom Chains," Mech. Mach. Theory, 19, pp. 507-530.

[12] Davies, T. H., and Crossly, F. E., 1966, "Structural Analysis of Plane Linkages by Franke's Condensed Notation,” J. Mech., 1, pp. 171-183.

[13] Freudenstein, F., and Maki, E. R., 1983, "Development of an Optimum Variable-Stroke Internal-Combustion Engine Mechanism From The Viewpoint of Kinematic Structure," ASME J. Mech., Transm., Autom. Des., 105, pp. 259-267.

[14] Freudenstein, F., and Maki, E. R., 1984, "Kinematic Structure of Mechanisms for Fixed and Variable-Stroke Axial-Piston Reciprocating Machines," ASME J. Mech., Transm., Autom. Des., 106, pp. 355-364.

[15] Datseris, P., and Palm, W., 1985, "Principles on the Development of Mechanical Hands Which Can Manipulate Objects by Means of Active Control," ASME J. Mech., Transm., Autom. Des., 107, pp. 148-156.

[16] Erdman, A. G., and Bowen, J., 1981, "Type and Dimensional Synthesis of Casement Window Mechanism,” Mech. Eng. (Am. Soc. Mech. Eng.), 103, pp. 46-55.

[17] Yan, H. S., 1992, “A Methodology for Creative Mechanism Design," Mech. Mach. Theory, 27, pp. 235-242.

[18] Tsai, L. W., 2000, Mechanism Design: Enumeration of Kinematic Structure According to Function, CRC Press, New York.

[19] Belfoire, N. P., and Tsai, L. W., 1991, "A New Methodology for Structural Synthesis of Geared Robotic Wrists," in Proceedings of the Second National Conference on Applied Mechanisms and Robotics, Paper No. VIB. 5.

[20] Zhou, H., and Ting, K. L., 2005, "Topological Synthesis of Compliant Mechanisms Using Spanning Tree Theory," ASME J. Mech. Des., 127, pp. 753-759.

[21] Pahl, G., and Beitz, W., 1999, Engineering Design: A Systematic Approach, Springer Verlag, New York.

[22] Suh, N. P., 1990, The Principle of Design, Oxford University Press, Oxford.

[23] Reuleaux, F., 1876, Kinematics of Machinery, McMillan, London. 